\title{
Surface- and pressure-induced bulk Kondo breakdown in $\mathrm{SmB}_{6}$
}

\author{
K. Chen, ${ }^{1, *}$ T-C. Weng, ${ }^{2}$ G. Schmerber, ${ }^{3}$ V. N. Gurin, ${ }^{4}$ J.-P. Kappler, ${ }^{1}{ }^{\text {Q. Kong, }},{ }^{1}$ F. Baudelet, ${ }^{1}$ A. Polian,,${ }^{1,5}$ and L. Nataf ${ }^{1}$ \\ ${ }^{1}$ Synchrotron SOLEIL, L'Orme des Merisiers, Saint-Aubin-BP48, 91192 Gif-sur-Yvette Cedex, France \\ ${ }^{2}$ Center for High Pressure Science \& Technology Advanced Research, 1690 Cailun Road, 201203 Shanghai, China \\ ${ }^{3}$ IPCMS, CNRS-Université de Strasbourg, 23 rue du Loess, BP 43, 67034 Strasbourg Cedex 2, France \\ ${ }^{4}$ Ioffe Institute, Russian Academy of Sciences, St. Petersburg 194021, Russia \\ ${ }^{5}$ IMPMC, Sorbonne Université, C.N.R.S. UMR 7590, 4 Place Jussieu, 75005 Paris, France
}

(Received 2 March 2018; revised manuscript received 13 June 2018; published 29 June 2018)

\begin{abstract}
Motivated by the prediction of surface Kondo breakdown in topological Kondo insulators, we investigated the valence and magnetism of $\mathrm{SmB}_{6}$ with x-ray absorption and magnetic circular dichroism at the $\operatorname{Sm} M_{4,5}$ and $L_{2}$ edges with surface and bulk sensitivity, respectively. A higher Sm valence state at the surface and in the bulk under pressure indicates the surface- and pressure-induced bulk Kondo breakdown. We confirmed the different magnetization origin from the surface ( $f$ electron) to the bulk ( $d$ electron) and ascertained the direct relationship between the disappearance of the $\mathrm{Sm} L_{2}$-edge $\mathrm{x}$-ray magnetic circular dichroism signal and the closure of the $d$ - $f$ hybridization gap in $\mathrm{SmB}_{6}$ bulk under pressure, paving the way for further investigations of the Kondo insulators. Above $P_{c}=8.5 \mathrm{GPa}$, a more localized $4 f$ state with higher valence and the disappearing of the $5 d$ magnetism are observed, suggesting the closure of the hybridization gap in the bulk.
\end{abstract}

DOI: 10.1103/PhysRevB.97.235153

\section{INTRODUCTION}

The Kondo insulator $\mathrm{SmB}_{6}$ was considered as a good candidate material of topological Kondo insulator [1-6]. The long-standing mysterious low-temperature-resistance plateau can be well explained with the topologically protected metallic surface states within a hybridization gap hosted in the topological Kondo insulator [7-14]. In $\mathrm{SmB}_{6}$, the strong hybridization of the low-lying localized $4 f$ electronic bands with a broad $5 d$ conduction band [15,16] via the Kondo effect gives rise to a hybridization gap of the order of $20 \mathrm{meV}$, resulting in the screening of localized $4 f$ magnetic moments. The strong hybridization also leads to a mixture of the $\mathrm{Sm}^{2+}$ $\left(4 f^{6}\right)$ and $\mathrm{Sm}^{3+}\left(4 f^{5}\right)$ configurations, with the electronic structure described by the Hund's rule ground states with total orbital momenta of $J=0$ and $5 / 2$, respectively. The bulk and surface electronic properties have been investigated using a wide range of experimental methods, highpressure x-ray-absorption spectroscopy experiments $[17,18]$, scanning tunneling spectroscopy [19-21], angle-resolved photoelectron spectroscopy [22-24], hard x-ray photoelectron spectroscopy [25,26], and resonant soft x-ray reflectometry [27] providing additional confirmation of surface states in $\mathrm{SmB}_{6}$.

Recently, a Kondo breakdown scenario was proposed based on the reduced screening of the local moments at the surface and the marked reduction in the surface Kondo temperature $\mathrm{T}_{K}^{S} \sim T_{K} / 10$ [28,29]. With the reduction of nearest-neighbor sites of the $\mathrm{Sm}^{3+}$ ions on the surface, the screening of local moments is either shifted to lower temperatures [7,8,10,21], or disappears completely [13]. A change in Sm valence at the

*kaichen.hzg@gmail.com surface has been reported from x-ray-absorption spectroscopy (XAS) and X-ray magnetic circular dichroism (XMCD), which is claimed to be the definitive proof of changes in the electronic structure at the surface of $\mathrm{SmB}_{6}$ [11]. A surface dominated conduction at $240 \mathrm{~K}$ has been achieved in epitaxial $\mathrm{SmB}_{6}$ films with a small $0.7 \%$ tensile strain enhanced $d-f$ hybridization [30], shedding light on the tuning of the ground state of the compound. For bulk $\mathrm{SmB}_{6}$, pressure-induced long-range magnetic ordering was reported in the metallic state above a critical pressure $P_{c}$ from nuclear forward scattering and specific-heat measurements [31], and the pressure-induced localization of $4 f$ states was supported by ${ }^{11} \mathrm{~B}-\mathrm{NMR}$ results [32].

Different magnetic properties are expected for the metallic surface and the insulator bulk of $\mathrm{SmB}_{6}$. Considering the surface Kondo breakdown scenario, the surface magnetism is mainly due to the $4 f$ electrons. On the contrary, as recently reported from inelastic neutron-scattering investigations, the $5 d$ magnetic contribution [33] is expected in the bulk due to the screening of the $4 f$ moments. Under pressure, the hybridization gap in bulk $\mathrm{SmB}_{6}$ will be closed, resulting in the more localized $4 f$ electrons and less $\mathrm{Sm}$ ions with $\mathrm{Sm}^{2+}$ configuration. To validate the proposed Kondo breakdown scenario, in this paper we report the surface and bulk electronic and magnetic properties of $\mathrm{SmB}_{6}$, investigated with XAS and XMCD at $\operatorname{Sm} M_{4,5}$ edges, and $\operatorname{Sm} L_{2}$ edge at $T=8 \mathrm{~K}$ with pressure up to $26 \mathrm{GPa}$. We observed the obvious $4 f$ magnetism with a higher valence of $v=2.74(3)(T=4 \mathrm{~K})$ at the surface and a very small $4 f$ bulk magnetism with the lower valence of $v=2.55(3)(T=8 \mathrm{~K})$. The $5 d$ magnetic contribution of bulk $\mathrm{SmB}_{6}$ is clearly observed from the $\mathrm{Sm} L_{2}$-edge XMCD spectra, and disappears around $\sim 7-10 \mathrm{GPa}$. These results confirmed the Kondo breakdown scenario in $\mathrm{SmB}_{6}$, accompanied by a reduced $d$ - $f$ hybridization in both cases. 


\section{SAMPLES AND EXPERIMENTAL SET-UP}

As a congruently melting compound [34-36], $\mathrm{SmB}_{6}$ has been prepared using the floating zone melting technique, by sealing a quartz ampoule with an excess of Sm, due to the higher pressure of $\mathrm{Sm}$ vapor than that of boron, under a pressurized gas atmosphere of pure Ar. Single phase $\mathrm{SmB}_{6}$ powder was characterized by laboratory $\mathrm{x}$-ray diffraction (XRD) measurement which leads to a lattice parameter $a=$ 4.1341(5) $\AA$, with an average size of the crystallites of the order of $200 \mathrm{~nm}$.

$\mathrm{SmB}_{6}$ powder was compressed into pellets for soft x-ray measurements. XAS and XMCD spectra at the $\mathrm{Sm} M_{4,5}$ edges were measured from 4 to $300 \mathrm{~K}$ using the surface sensitive total electron yield (TEY) and bulk sensitive fluorescence yield (FY) mode with a normal incidence configuration. However, the "surface" defined here refers to the electron escaping length of the order of $\sim 2-5 \mathrm{~nm}$ while the "bulk" is related to a thickness 10 times higher. The XMCD spectra were carried out using $\mathrm{x}$ rays of circular right (CR) and left (CL) polarizations with a magnetic field of $\mu_{0} H= \pm 6.5 \mathrm{~T}$ applied along the beam, recorded as $\sigma^{+}-\sigma^{-}$, where $\sigma^{+}$and $\sigma^{-}$denote the absorption cross section for right and left circular polarized $\mathrm{x}$ rays. A self-absorption correction procedure has been made based on the Fluo algorithm in the ATHENA analysis software [37] for the bulk XAS and XMCD spectra recorded FY-XAS. The XAS and XMCD spectra at the $\mathrm{Sm} L_{2}$ - edge have been performed to probe the pressure-dependent local electronic configuration and $5 d$ electron magnetism of $\mathrm{Sm}$ atoms. $\mathrm{SmB}_{6}$ powder, together with the pressure transmitting medium silicon oil, was pressurized up to $26 \mathrm{GPa}$ in a diamond-anvil cell (DAC). The pressure was measured using the ruby fluorescence scale. XMCD was obtained through the difference of XAS spectra measured under a magnetic field of $\mu_{0} H=1.3 \mathrm{~T}$ applied parallel or antiparallel to the beam helicity. The spectra of Sm $M_{4,5}$ and $L_{2}$ edge were recorded at the DEIMOS [38] and ODE beamlines [39] respectively, at synchrotron SOLEIL, France.

\section{RESULTS AND DISCUSSION}

The XAS spectra of Sm $M_{4,5}$ edges, collected simultaneously using TEY and FY modes at $T=4 \mathrm{~K}$, are shown in Fig. 1(a). The Sm $3 d-4 f$ transition is split into a $3 d_{5 / 2}$ and a $3 d_{3 / 2}$ branch due to the spin-orbit interaction. Each of these branches is a mixture of the $\mathrm{Sm}^{2+}\left(4 f^{6}\right)$ and $\mathrm{Sm}^{3+}\left(4 f^{5}\right)$ components. The shoulder and main peak positions of the Sm $M_{5}$ edge, addressed to be the contribution from $\mathrm{Sm}^{2+}$ and $\mathrm{Sm}^{3+}$, are well reproduced from atomic multiplet simulation using CTM4XAS code [40], with the electrostatic and exchange parameters scaled down to $80 \%$ of the atomic Hartree-Fock value, and convoluted with a Lorentzian function for lifetime broadening and a Gaussian to account for the instrumental resolution. The shoulder in the bulk spectra is stronger than that in the surface spectra, indicating a higher $\mathrm{Sm}^{2+}$ contribution and a lower Sm valence state. We investigate whether the spectrum can be interpreted using the spectra of $\mathrm{Sm}^{2+}$ and $\mathrm{Sm}^{3+}$ from the full multiplet simulation shown in Fig. 1(a). A weighted sum of TEY- and FY-XAS (4 K) has been built and compared to the experimental spectra, shown in Figs. 1(b) and 1(c). The weighted sum $\left(27 \% \mathrm{Sm}^{2+}\right.$ and $73 \% \mathrm{Sm}^{3+}$ for surface,
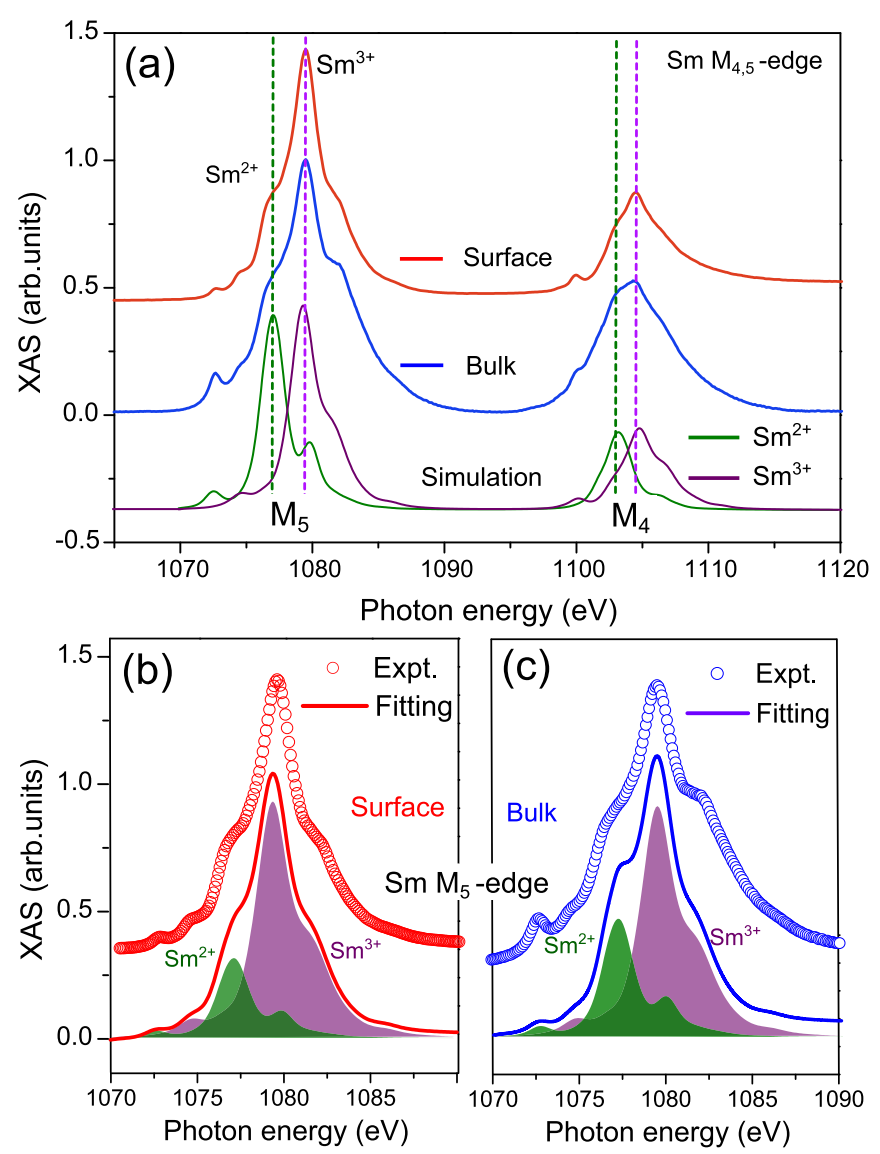

FIG. 1. (a) XAS of $\mathrm{SmB}_{6}$ at $\mathrm{Sm} M_{4,5}$ edges of TEY (red curve) and FY-XAS (blue curve) at $T=4 \mathrm{~K}$, as well as the spectra of $\mathrm{Sm}^{2+}$ and $\mathrm{Sm}^{3+}$ from atomic multiplet calculation. The surface (b) and bulk (c) XAS spectra can be well fitted with the combination of the spectra of $\mathrm{Sm}^{2+}$ and $\mathrm{Sm}^{3+}$ from atomic multiplet calculation.

and $35 \% \mathrm{Sm}^{2+}$ and $65 \% \mathrm{Sm}^{3+}$ for bulk) of the simulated curves describe the $\mathrm{SmB}_{6}$ spectrum very well. The $\mathrm{Sm}$ mean valence in $\mathrm{SmB}_{6}$ can be derived from $v=2+I\left(f^{5}\right) /\left[I\left(f^{5}\right)+I\left(f^{6}\right)\right]$, where $I\left(f^{5}\right)$ and $I\left(f^{6}\right)$ denote the integrated intensities of the $\mathrm{Sm}^{3+}$ and $\mathrm{Sm}^{2+}$ components. The deduced $\mathrm{Sm}$ valences are 2.73(3) and 2.65(5) for the surface and bulk $\mathrm{SmB}_{6}$, respectively. Zabolotnyy et al., reported that the highly polar (001) surface undergoes substantial chemical and valence reconstruction, resulting a $\mathrm{Sm}^{3+}$ dominated subsurface region [27]. Here we have small particles (several micrometers) that give a rather rough surface mixed with different crystal surfaces. Our results indicate that the surface valence reconstruction may be formed on all surfaces of $\mathrm{SmB}_{6}$.

Even after the self-absorption correction for the FY XAS, the valence of $\mathrm{Sm}$ still can be overestimated [41]. In order to extract the valence of the $\mathrm{Sm}$ in the bulk under pressure, experimental $\mathrm{Sm} L_{2}$ edge XAS spectra are recorded and shown in Fig. 2(a) $(T=8 \mathrm{~K}, P=0.6-26 \mathrm{GPa})$. The $\mathrm{Sm}^{2+}$ contribution, marked with a dashed line as the shoulder in Fig. 2(a), decreases with pressure which indicates the increase of the $\mathrm{Sm}$ valence. The spectra are analyzed by assigning identical line shapes (with core hole lifetime broadening) to the $\mathrm{Sm}^{2+}$ and $\mathrm{Sm}^{3+}$ contribution in the spectra, each with a proportionality tanh-type background, as shown in Figs. 2(b) 


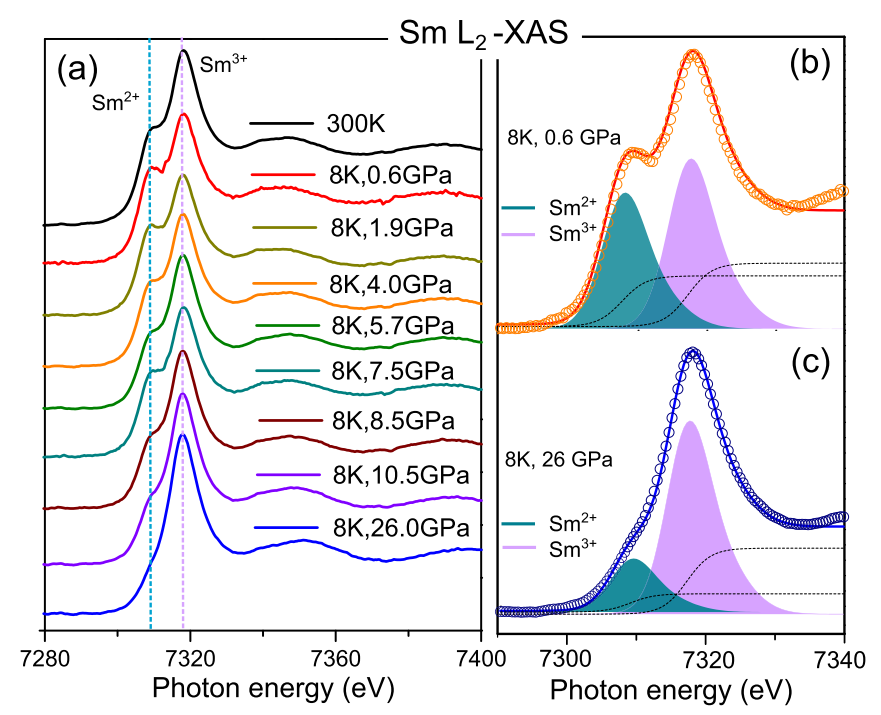

FIG. 2. (a) XAS of $\mathrm{Sm} L_{2}$ edges at $8 \mathrm{~K}$ under pressure up to $26 \mathrm{GPa}$, and the spectrum recorded at $300 \mathrm{~K}$ for comparison. The $L_{2}$ edge XAS at $8 \mathrm{~K}, P=0.6$ (b) and $26.0 \mathrm{GPa}$ (c) were well fitted with the combination of the spectra of $\mathrm{Sm}^{2+}$ and $\mathrm{Sm}^{3+}$ with identical line shapes, with the tanh-type background.

and 2(c) for pressure of 0.6 and 26.0 GPa, respectively. Derived from the fitting procedure, the $\mathrm{Sm}$ ion has a lower mean valence of $v=2.55(3)$ at $T=8 \mathrm{~K}$ and $P=0.6 \mathrm{GPa}$ and a much higher mean valence of $v=2.78(3)$ when the pressure increased up to $26.0 \mathrm{GPa}$. This bulk Sm valence under pressure $(v=2.78)$ is even higher than the surface Sm valence $(v=2.73)$ at ambient pressure determined from $\mathrm{Sm} M_{4,5}$ XAS.

Applying the fitting procedure, we extracted the Sm mean valence as a function of temperature and pressure (Fig. 3). As shown in the inset, the mean valence of surface $\mathrm{Sm}$ ions is higher compared to the value of the bulk Sm ions through the

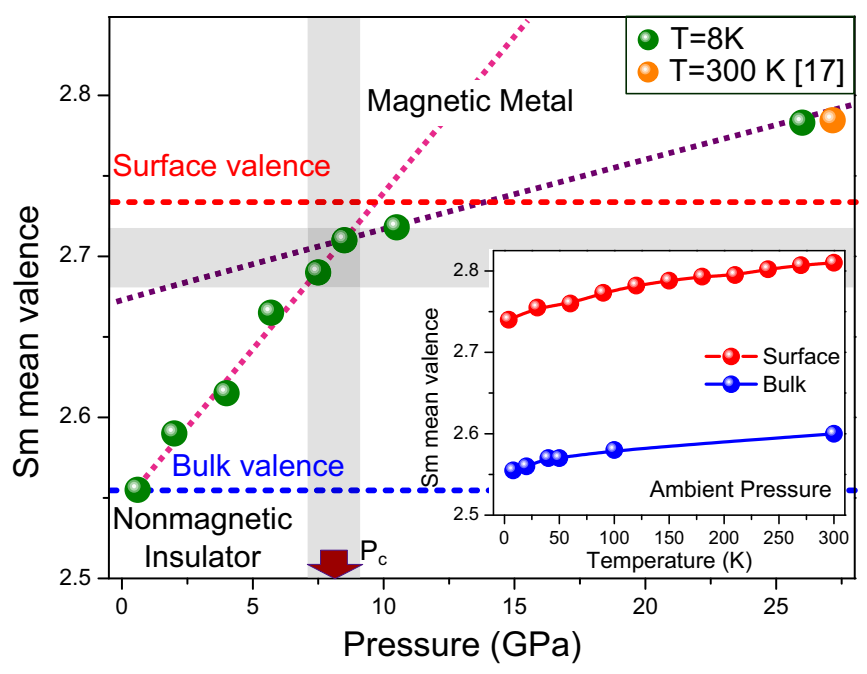

FIG. 3. Pressure dependence of the $\mathrm{Sm}$ mean valence in $\mathrm{SmB}_{6}$. The inset shows the temperature dependence of the surface and bulk $\mathrm{Sm}$ mean valence in $\mathrm{SmB}_{6}$, respectively. The grey area shows the region of nonmagnetic insulator to magnetic metal transition. Error bars are smaller than the points. whole temperature range, and both of them increase gradually with temperature. At ambient pressure, the valence of the Sm ions changes slightly from $v=2.55(3)$ to 2.60(3) in bulk and from $v=2.73$ (3) to 2.80 (3) on surfaces from $T=4$ to $300 \mathrm{~K}$. In general, our findings for the bulk Sm valence and its temperature dependence are consistent with the results reported in earlier Sm $L_{2,3}$-edge XAS experiments [42]. A relative higher valence state $(v=2.73)$ is obtained at the surface, consistent with the surface Kondo breakdown scenario, in contrast to the entire surface $\mathrm{Sm}^{3+}$ reported in [11]. At $T=8 \mathrm{~K}$, a quick enhancement of the valence was observed under pressure from $v=2.55$ at $0.6 \mathrm{GPa}$ to $v=2.70$ at $P_{c}=\sim 8 \mathrm{GPa}$, followed by a slow valence increase to $v=2.78$ at $26 \mathrm{GPa}$, across the level of the surface valence value of $v=2.73$. $\mathrm{SmB}_{6}$ becomes magnetically ordered below $12 \mathrm{~K}[31,43]$ once the insulating gap closes by 5-10 GPa $[44,45]$, with a Sm mean valence of $v=\sim 2.69(3)$ [17] (marked as the grey area in Fig. 3). In the Kondo breakdown regime above $\sim 10 \mathrm{GPa}$, the $\mathrm{Sm}$ valence remains unchanged with temperature in bulk $\mathrm{SmB}_{6}$. When comparing the present experimental results $(8 \mathrm{~K})$ with the previous data recorded at $300 \mathrm{~K}$ [17] at $\sim 26 \mathrm{GPa}$, we found that the Sm valence remains unchanged, consistent with the pressure-induced Kondo breakdown scenario.

The higher Sm mean valence indicates a relative weaker hybridization between $4 f$ and $5 d$ states on the $\mathrm{SmB}_{6}$ surface than in the bulk at ambient pressure, and a weaker hybridization in the bulk under pressure. In both cases, the screening of $4 f$ local moments will be suppressed. This will make the $4 f$ magnetization the main magnetic contribution at the surface or in the bulk under pressure. In the bulk at ambient pressure, the local $4 f$ magnetization is highly screened by the conduction electrons, resulting in the paramagnetic ground state. Hence the magnetic contributions from the $5 d$ electrons is not negligible. The $5 d$ magnetization as well as the reduced $4 f$ magnetization in bulk $\mathrm{SmB}_{6}$ were observed from the XAS and XMCD of Sm at $L_{2}$ edge and $M_{4,5}$ edge.

Based on the surface Kondo breakdown scenario, the surface magnetization is expected to be much stronger compared to the bulk with normal Kondo screening of $4 f$ magnetization. To confirm the proposed reduction of Kondo screening at the surface in more detail, we collected Sm $M_{4,5}$-edge XMCD spectra simultaneously for surface (red curve) and bulk (blue curve) at $T=4 \mathrm{~K}$ and $\mu_{0} H=6.5 \mathrm{~T}$ [Fig. 4(a) inset]. According to the nonmagnetic $J=0$ singlet $\left(4 f^{6}\right)$ of $\mathrm{Sm}^{2+}$, the XMCD signal comes from the $\mathrm{Sm}^{3+}$ contribution at the surface with a shape similar to that observed in $\mathrm{SmAl}_{2}$ [46] and $\mathrm{Sm}_{0.974} \mathrm{Gd}_{0.02} \mathrm{Al}_{2}$ [47]. Several phenomena related to the multiplet structure of the intermediate state may occur which render FY XMCD different from the true XMCD spectrum at the $M_{4,5}$ edge of rare earths [41]. Thus, the main qualitative observation for the bulk is a reduced XMCD intensity, roughly $1 / 4$, as compared to the XMCD on the surface, indicating a much smaller $4 f$ contributed magnetization in the bulk $\mathrm{SmB}_{6}$. Besides, the bulk XMCD spectrum from the FY mode shows a negative signal at the $\mathrm{Sm} M_{4}$ edge, in contrast with the positive signal at the surface. Those results state clearly that the $4 f$ magnetic contribution, which dominates the surface magnetism, is strongly reduced in the bulk.

Sm $L_{2}$-edge XAS and XMCD spectra are presented in Fig. 4(b), recorded at $40 \mathrm{~K}, P=0.6 \mathrm{GPa}$, and $\mu_{0} H=1.3 \mathrm{~T}$. 


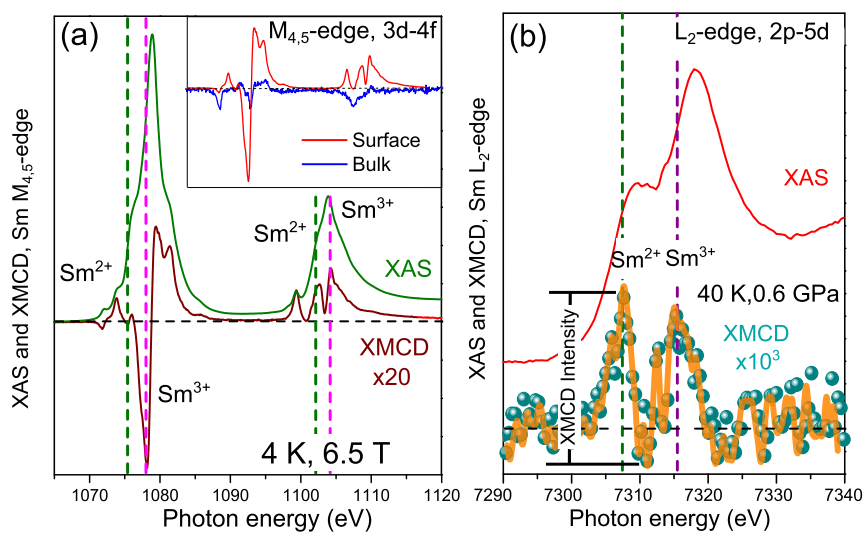

FIG. 4. (a) XAS and XMCD of $\mathrm{Sm}$ at $\mathrm{SmB}_{6}$ surface at $M_{4,5}$ edge $(3 d-4 f)$ at $T=4 \mathrm{~K}, 6.5 \mathrm{~T}$, ambient pressure, and (b) $L_{2}$ edge ( $2 p-5 d$ ) at $8 \mathrm{~K}, 1.3 \mathrm{~T}, 0.6 \mathrm{GPa}$ for bulk $\mathrm{SmB}_{6}$. The comparison of the $\mathrm{Sm} M_{4,5}$ XMCD spectra from TEY XAS (red curve) and FY XAS (blue curve) is shown as inset in (a).

Two well defined peaks are clearly present in both XAS and $\mathrm{XMCD}$ spectra. The quadrupolar contribution as a result of $4 f / 5 d$ hybridization is strong in $\mathrm{Sm} L_{3}$ edge but highly reduced in $L_{2}$ edge XMCD spectra in $\mathrm{SmN}$ [48]. Thus these spectral features with separated peaks from $\mathrm{Sm}^{2+}$ and $\mathrm{Sm}^{3+}$ in the XANES and XMCD signal were related to the dipolar contribution from the $2 p-5 d$ excitations. The peak positions of the XMCD spectra are several eV below the XANES peaks, similar to that observed in $\mathrm{Sm}_{1-x} \mathrm{Gd}_{x} \mathrm{Al}_{2}$ [49].

The magnetization curve of bulk $\mathrm{SmB}_{6}$ as a function of temperature measured with a magnetometer property measurement system superconducting quantum interference device vibrating-sample magnetometer (Quantum Design) is shown in Fig. 5(a), under zero-field-cooling (ZFC) or field-cooling (FC) conditions in an applied field of $\mu_{0} H=2 \mathrm{~T}$. The bulk magnetization value is in good agreement with Ref. [11].
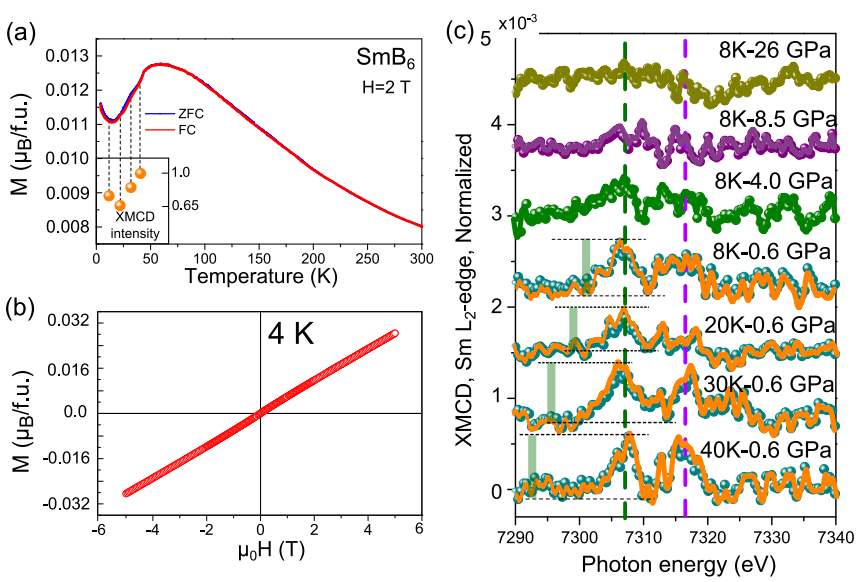

FIG. 5. (a) Magnetization and (b) paramagnetic magnetic curve $M(H)$ loop at $4 \mathrm{~K}$. (c) Temperature- and pressure-dependent $\mathrm{Sm} L_{2}$ edge XMCD spectra of bulk $\mathrm{SmB}_{6}$, normalized to the XAS intensity. This $L_{2}$-edge XMCD intensity follows the temperature behavior of the bulk magnetization as shown in (a) inset, and disappears when $P>P_{c} \sim 8.5 \mathrm{GPa}$.
The paramagnetic curve with no observable hysteresis in the $M(H)$ loop of $\mathrm{SmB}_{6}$ taken at $T=4 \mathrm{~K}$ is shown in Fig. 5(b). The temperature- and pressure-dependent $\operatorname{Sm} L_{2}$-edge XMCD spectra of bulk $\mathrm{SmB}_{6}$ are shown in Fig. 5(c), normalized to the XAS intensity. The bulk magnetism shows a maximum around $50 \mathrm{~K}$ and a dip at around $15 \mathrm{~K}$ [Fig. 5(a)]. The normalized temperature-dependent intensity of the Sm $L_{2}$ edge XMCD spectra follows the similar temperature behavior as the bulk magnetization, as shown in Fig. 5(a) inset. It indicates the direct relationship between the $5 d$ electrons and the bulk paramagnetic magnetization in $\mathrm{SmB}_{6}$ at ambient pressure. At fixed temperature of $8 \mathrm{~K}$, the intensity of the XMCD spectra decreases under pressure and disappears when $P>P_{c} \sim 8.5 \mathrm{GPa}$.

The $\mathrm{SmB}_{6}$ surface magnetism can be estimated, based on the enhanced XMCD spectra shown in Fig. 4(a), to compare with the bulk magnetization curve in Figs. 5(a) and 5(b). The XMCD sum rules can yield separate values of $M_{S z}$ and $\mathrm{M}_{L z}$ based on the integration of the XAS and XMCD spectra, with a correction factor of $X_{I} / X_{E}=3$ of the spin sum rule due to the $j j$ mixing of the two spin-orbit split states of $\mathrm{Sm}^{3+}$ $[46,47,50]$. We obtain the orbital and spin moments, $M_{L}=$ $0.10(2) \mu_{B}$ and $M_{S}=-0.03(1) \mu_{B}$, which lead to a magnetic moment at the surface of $0.07(3) \mu_{B}$, at $T=4 \mathrm{~K}$ and $\mu_{0} H=$ $6.5 \mathrm{~T}$, compared to the bulk magnetization of $\sim 0.035(5) \mu_{B}$ shown in Fig. 5(b) (extrapolated to 6.5 T). The surface magnetic moment is twice enhanced compared to the bulk magnetism. Since the sum rule is not valid for the FY-mode XMCD spectra, the reduced intensity indicates that the $4 f$ states bear a small magnetic moment in the bulk.

The highly reduced $4 f$ magnetization indicates that the bulk magnetization of $0.035(5) \mu_{B}$ mainly comes from the $5 d$ electron contribution, which is parallel to the applied magnetic field. Considering the ferromagnetic coupling between the spin magnetic moments of $4 f$ and $5 d$ electrons, the $4 f$ spin in bulk $\mathrm{SmB}_{6}$ is parallel aligned along the magnetic field. In contrast, at the surface, the $4 f$ orbital and spin moments are parallel and antiparallel to the magnetic field, respectively. The $\operatorname{Sm} 4 f$ spin magnetization changes its direction from the surface to the bulk, as observed in Fig. 4(a) inset, with opposite sign of the XMCD spectra from the surface and bulk using TEY and FY modes, respectively. Pressure-induced long-range magnetic ordering as well as the localization of Sm $4 f$ electrons have been expected from the pressure-induced Kondo breakdown scenario in $\mathrm{SmB}_{6}$ bulk. In this scenario, the magnetization in the bulk is dominated by the more localized $4 f$ electrons while the contribution from the $5 d$ electrons is reduced from the closed hybridization gap under pressure. The $4 f-5 d$ hybridization in $\mathrm{SmB}_{6}$ under pressure is directly related to the $\mathrm{Sm}$ $L_{2}$-edge XMCD spectra, similar to the relationship observed in uranium compounds $\left(\mathrm{UCu}_{2} \mathrm{Si}_{2}\right.$ and $\left.\mathrm{UMn}_{2} \mathrm{Si}_{2}\right)$ between the $5 f-6 d$ hybridization and the $\mathrm{U} L_{3}$-edge XMCD spectra [51]. Besides, a magnetically ordered state with a saturated moment of $0.5 \mu_{B}$ and an ordering temperature of $12 \mathrm{~K}$ was observed from high-pressure ${ }^{149} \mathrm{Sm}$ nuclear forward scattering up to $26 \mathrm{GPa}$ [31]. The vanishing XMCD singal above $8 \mathrm{GPa}$ here indicates that the magnetic order of $\mathrm{SmB}_{6}$ should be of the antiferromagnetic type with no net Sm magnetization, since the ferromagnetic ordering of Sm $4 f$ moments will results in $5 d$ magnetization via intra-atomic exchange and this 
ferromagnetism scenario can be ruled out by our experimental results.

\section{CONCLUSION}

We conclude that the recently proposed surface- and pressure-induced bulk Kondo breakdown in Kondo insulator $\mathrm{SmB}_{6}$ are confirmed by XAS and XMCD spectra at the Sm $M_{4,5}$ edge and $L_{2}$ edge. Due to the reduced Kondo screening, as well as the $d$ - $f$ hybridization, Sm ions at the surface have higher valence states and strongly enhanced magnetization of $4 f$ states compared to the $\mathrm{Sm}$ ions in the bulk. A transition from nonmagnetic insulator to magnetic metal has been observed with the Sm valence increase up to $v=2.78$ at $26 \mathrm{GPa}$ at $T=8 \mathrm{~K}$. Bulk Sm ions lose their $5 d$ electron magnetization under pressure for $P>P_{c}=8.5 \mathrm{GPa}$, entering the long-range magnetic ordering state dominated by the localized $4 f$ magnetization. We observed the direct relationship between the disappearance of the $\operatorname{Sm~} L_{2}$-edge XMCD signal and the closed $d-f$ hybridization gap in $\mathrm{SmB}_{6}$ bulk under pressure, demonstrating the potentiality of this methodology to investigate other Kondo insulators.

\section{ACKNOWLEDGMENT}

We acknowledge Synchrotron-Soleil for provision of synchrotron radiation facilities.
[1] M. Dzero, K. Sun, V. Galitski, and P. Coleman, Phys. Rev. Lett. 104, 106408 (2010).

[2] T. Takimoto, J. Phys. Soc. Jpn. 80, 123710 (2011).

[3] M. Dzero, K. Sun, P. Coleman, and V. Galitski, Phys. Rev. B 85, 045130 (2012).

[4] F. Lu, J. Z. Zhao, H. Weng, Z. Fang, and X. Dai, Phys. Rev. Lett. 110, 096401 (2013).

[5] M. Dzero and V. Galitski, J. Exp. Theor. Phys. 117, 499 (2013).

[6] V. Alexandrov, M. Dzero, and P. Coleman, Phys. Rev. Lett. 111, 226403 (2013).

[7] D. J. Kim, S. Thomas, T. Grant, J. Botimer, Z. Fisk, and J. Xia, Sci. Rep. 3, 3150 (2013).

[8] S. Wolgast, C. Kurdak, K. Sun, J. W. Allen, D.-J. Kim, and Z. Fisk, Phys. Rev. B 88, 180405(R) (2013).

[9] X. Zhang, N. P. Butch, P. Syers, S. Ziemak, R. L. Greene, and J. Paglione, Phys. Rev. X 3, 011011 (2013).

[10] D. J. Kim, J. Xia, and Z. Fisk, Nat. Mater. 13, 466 (2014).

[11] W. A. Phelan, S. M. Koohpayeh, P. Cottingham, J. W. Freeland, J. C. Leiner, C. L. Broholm, and T. M. McQueen, Phys. Rev. X 4, 031012 (2014).

[12] S. Thomas, D. J. Kim, S. B. Chung, T. Grant, Z. Fisk, and J. Xia, Phys. Rev. B 94, 205114 (2016).

[13] Y. Nakajima, P. Syers, X. Wang, R. Wang, and J. Paglione, Nat. Phys. 12, 213 (2016).

[14] Q. Wu and L. Sun, Rep. Prog. Phys. 80, 112501 (2017).

[15] G. Aeppli and Z. Fisk, Comments Condens. Matter Phys. 16, 155 (1992).

[16] P. W. Anderson, Adv. Phys. 49, 257 (2000).

[17] N. P. Butch, J. Paglione, P. Chow, Y. Xiao, C. A. Marianetti, C. H. Booth, and J. R. Jeffries, Phys. Rev. Lett. 116, 156401 (2016).

[18] L. Sun and Q. Wu, Rep. Prog. Phys. 79, 084503 (2016).

[19] S. Rö $\beta$ ler, T.-H. Jang, D.-J. Kim, L. H. Tjeng, Z. Fisk, F. Steglich, and S. Wirth, Proc. Natl. Acad. Sci. USA 111, 4798 (2014).

[20] W. Ruan, C. Ye, M. Guo, F. Chen, X. Chen, G. M. Zhang, and Y. Wang, Phys. Rev. Lett. 112, 136401 (2014).

[21] L. Jiao, S. Rö $\beta$ ler, D.-J. Kim, L. H. Tjeng, Z. Fisk, F. Steglich, and S. Wirth, Nat. Commun. 7, 13762 (2016).

[22] Z.-H. Zhu, A. Nicolaou, G. Levy, N. P. Butch, P. Syers, X. F. Wang, J. Paglione, G. A. Sawatzky, I. S. Elfimov, and A. Damascelli, Phys. Rev. Lett. 111, 216402 (2013).

[23] M. Neupane, N. Alidoust, S.-Y. Xu, T. Kondo, Y. Ishida, D.-J. Kim, C. Liu, I. Belopolski, Y. J. Jo, T.-R. Chang, H.-T. Jeng, T.
Durakiewicz, L. Balicas, H. Lin, A. Bansil, S. Shin, Z. Fisk, and M. Z. Hasan, Nat. Commun. 4, 2991 (2013).

[24] J. Jiang, S. Li, T. Zhang, Z. Sun, F. Chen, Z. R. Ye, M. Xu, Q. Q. Ge, S. Y. Tan, X. H. Niu, M. Xia, B. P. Xie, Y. F. Li, X. H. Chen, H. H. Wen, and D. L. Feng, Nat. Commun. 4, 3010 (2013).

[25] P. Lutz, M. Thees, T. R. F. Peixoto, B. Y. Kang, B. K. Cho, C. H. Min, and F. Reinert, Philos. Mag. 96, 3307 (2016).

[26] Y. Utsumi, D. Kasinathan, K.-T. Ko, S. Agrestini, M. W. Haverkort, S. Wirth, Y.-H. Wu, K.-D. Tsuei, D.-J. Kim, Z. Fisk, A. Tanaka, P. Thalmeier, and L. H. Tjeng, Phys. Rev. B 96, 155130 (2017).

[27] V. B. Zabolotnyy, K. Fürsich, R. J. Green, P. Lutz, K. Treiber, C.-H. Min, A. V. Dukhnenko, N. Y. Shitsevalova, V. B. Filipov, B. Y. Kang, B. K. Cho, R. Sutarto, F. He, F. Reinert, D. S. Inosov, and V. Hinkov, Phys. Rev. B 97, 205416 (2018).

[28] V. Alexandrov, P. Coleman, and O. Erten, Phys. Rev. Lett. 114, 177202 (2015).

[29] O. Erten, P. Ghaemi, and P. Coleman, Phys. Rev. Lett. 116, 046403 (2016).

[30] A. Stern, M. Dzero, V. M. Galitski, Z. Fisk, and J. Xia, Nat. Mater. 16, 708 (2017).

[31] A. Barla, J. Derr, J. P. Sanchez, B. Salce, G. Lapertot, B. P. Doyle, R. Rüffer, R. Lengsdorf, M. M. Abd-Elmeguid, and J. Flouquet, Phys. Rev. Lett. 94, 166401 (2005).

[32] K. Nishiyama, T. Mito, G. Pristas, Y. Jara, T. Koyama, K. Ueda, T. Kohara, Y. Akahama, S. Gabani, M. Reiffers, K. Flachbart, H. Fukazawa, Y. Kohori, N. Takeshita, and N. Shitsevalova, J. Phys. Soc. Jpn. 82, 123707 (2013).

[33] W. T. Fuhrman, J. Leiner, P. Nikolić, G. E. Granroth, M. B. Stone, M. D. Lumsden, L. DeBeer-Schmitt, P. A. Alekseev, J.-M. Mignot, S. M. Koohpayeh, P. Cottingham, W. A. Phelan, L. Schoop, T. M. McQueen, and C. Broholm, Phys. Rev. Lett. 114, 036401 (2015).

[34] V. N. Gurin and M. M. Korsukova, Prog. Cryst. Growth Charact. 6, 59 (1983).

[35] V. N. Gurin and L. I. Derkachenko, Progr. Cryst. Growth Charact. Mater. 27, 163 (1993).

[36] W. G. Moffatt, Handbook of Binary Phase Diagrams, edited by J. H. Westbrook (Genium Publ., Amsterdam, New York, 2006).

[37] B. Ravel and M. Newville, J. Synchrotron Radiat. 12, 537 (2005).

[38] P. Ohresser, E. Otero, F. Choueikani, K. Chen, S. Stanescu, F. Deschamps, T. Moreno, F. Polack, B. Lagarde, J.-P. Daguerre, 
F. Marteau, F. Scheurer, L. Joly, J.-P. Kappler, B. Muller, O. Bunau, and Ph. Sainctavit, Rev. Sci. Instrum. 85, 013106 (2014).

[39] F. Baudelet, L. Nataf, and R. Torchio, High Pres. Res. 36, 429 (2016).

[40] E. Stavitski and F. M. F. de Groot, Micron 41, 687 (2010).

[41] M. Pompa, A. M. Flank, P. Lagarde, J. C. Rife, I. Stekhin, M. Nakazawa, H. Ogasawara, and A. Kotani, Phys. Rev. B 56, 2267 (1997).

[42] M. Mizumaki, S. Tsutsui, and F. Iga, J. Phys.: Conf. Ser. 176, 012034 (2009).

[43] J. Derr, G. Knebel, G. Lapertot, B. Salce, M.-A. Méasson, and J. Flouquet, J. Phys. Condens. Matter 18, 2089 (2006).

[44] J. C. Cooley, M. C. Aronson, Z. Fisk, and P. C. Canfield, Phys. Rev. Lett. 74, 1629 (1995).
[45] J. Derr, G. Knebel, D. Braithwaite, B. Salce, J. Flouquet, K. Flachbart, S. Gabáni, and N. Shitsevalova, Phys. Rev. B 77, 193107 (2008).

[46] S. S. Dhesi, P. Bencok, N. B. Brookes, G. van der Laan, and R. M. Galera, J. Appl. Phys. 93, 8337 (2003).

[47] S. S. Dhesi, G. van der Laan, P. Bencok, N. B. Brookes, R. M. Galera, and P. Ohresser, Phys. Rev. B 82, 180402 (2010).

[48] E.-M. Anton, B. J. Ruck, C. Meyer, F. Natali, H. Warring, F. Wilhelm, A. Rogalev, V. N. Antonov, and H. J. Trodahl, Phys. Rev. B 87, 134414 (2013).

[49] M. Bersweiler, K. Dumesnil, F. Wilhelm, and A. Rogalev, Phys. Rev. B 88, 054411 (2013).

[50] T. Jo, J. Electron Spectrosc. Relat. Phenom. 86, 73 (1997).

[51] R. D. dos Reis, L. S. I. Veiga, C. A. Escanhoela Jr., J. C. Lang, Y. Joly, F. G. Gandra, D. Haskel, and N. M. Souza-Neto, Nat. Commun. 8, 1203 (2017). 